\title{
Günther Maihold
}

\section{Außenwissenschaftspolitik, diplomacia científica y relaciones internacionales científicas: Alemania-América Latina}

Außenwissenschaftspolitik es un neologismo cuya carrera política se ha venido incrementando en las relaciones exteriores de Alemania durante la última década. La "invención" del término tiene su origen en una propuesta del entonces secretario general de la Fundación Alexander von Humboldt, el Dr. Georg Schütte, quien enfatizaba que la diplomacia científica debería considerarse como uno de los ejes centrales de la acción externa de Alemania (Schütte 2006). Con este impulso al debate, Schütte trataba de llamar la atención sobre una creciente pérdida de presencia de las ciencias ubicadas en Alemania frente a una competencia internacional en aumento, y concluía que se debía a la falta de atracción de Alemania como lugar de estudio para los investigadores internacionales. Su motivación consistía en convencer a los actores políticos para que asumieran acciones decididas para tratar de orientar las corrientes internacionales de investigadores hacia Alemania y así fortalecer las capacidades de rendimiento científico en el país, inducir nuevas decisiones de cooperación científica entre universidades e institutos de investigación e incentivar inversiones de empresas multinacionales. Era ésta la forma en la que la Außenwissenschaftspolitik debía orientar la dimensión estratégica de la política científica de Alemania (Schütte 2008: 17). Este impulso, que emanó de la Fundación Alexander von Humboldt, ha sido asumido con gran entusiasmo por parte de la política alemana más allá de las líneas divisorias que tradicionalmente separan a los ministerios correspondientes. Tanto el Ministerio de Relaciones Exteriores (Steinmeier 2008) como el de Educación e Investigación (Schavan 2008) se identificaron con el llamado a relanzar el posicionamiento alemán en materia científica en el ámbito internacional, desarrollando programas específicos para alimentar esta presencia con proyectos y programas especiales.

Detrás del argumento de tratar de asumir la internacionalización de las ciencias como reto político se esconde, a veces, la ilusión de que el mismo proceso de la globalización implica un automatismo de internacionalización, al dejar a un lado la necesidad de organizar deliberadamente este proceso (Nettelbeck 2008). Allí descansa también un debate internacional que se orienta en 
dos direcciones: por un lado enfatiza que las metas de la ciencia también son parte de la política exterior, y confronta así esta posición con una alternativa que considera que los objetivos de la política exterior los efectúa la ciencia (The Royal Society 2010)

\section{La ubicación del tema en el ámbito administrativo alemán}

En términos generales, estas dos opciones describen diferentes situaciones en la relación entre política y ciencia: en la primera versión se pone de manifiesto que es la misma ciencia la que logra concebir sus propios objetivos y transmitirlos como metas generalizadas de la política en su acción externa, utilizando los instrumentos políticos establecidos por los Ministerios de Relaciones Exteriores para ir fortaleciendo su propia posición en el ámbito internacional. En cambio la segunda posición refleja una subordinación de la ciencia a los objetivos definidos por parte de la política exterior, y se ofrece como un instrumento para darles una mejor difusión y profundización en el ámbito internacional.

Aunque en esta presentación se expongan las dos opciones como contrapuestas, en la práctica probablemente no se perciba con tanta nitidez dicha confrontación; más bien se pueden señalar diferentes etapas en un proceso de configuración de una interdependencia existente entre los dos espacios, en la que se realza el posicionamiento de Alemania y su investigación académica en el mundo. Esto se realiza especialmente en el caso de un país sin muchos recursos naturales que busca conquistar mercados con base en sus adelantos de investigación y desarrollo tecnológico, motivo que llevó al respectivo Ministerio alemán de Educación e Investigación (BMBF) a lanzar en el año 2008 su "Iniciativa para la Internacionalización de la Ciencia y la Investigación" (BMBF 2008). Sin embargo, este esfuerzo no se limitó a esta dependencia gubernamental, sino que contó también con la emulación (para algunos observadores, la competencia) por parte del Ministerio de Relaciones Exteriores, que desarrolló su propio programa de fomento de las ciencias alemanes en el exterior. Llama la atención aquí el énfasis tan desigual que le pusieron los respectivos ministerios a sus iniciativas : mientras el Ministerio de Educación e Investigación adoptó para su punto de vista el concepto de una "carrera por el conocimiento" realzando la competencia internacional por las "cabezas pensantes" y la participación más activa de Alemania en esta competición, el Ministerio de Relaciones Exteriores intentó posicionar sus programas con un matiz de mayor cooperación bajo el título "Conectar los mundos del conocimiento" (Auswärtiges Amt 2009a y b). Así, la iniciativa del Ministerio de Educación e Investigación, con la inclusión de un grupo específico de países, lanza 
una orientación adicional y apunta hacia los "líderes globales" para fortalecer la cooperación en materia de investigación, logrando comprometer a los centros de Investigación y Desarrollo (I+D) para conformar alianzas que potencien la creatividad, pero al mismo tiempo establece formatos de cooperación de largo alcance en materia de educación superior e I+D con países en vías de desarrollo (Flink/Schreiterer/Turekian 2010). Todo esto se maneja con el afán de resolver retos de carácter global y para demostrar conjuntamente responsabilidad internacional. Es en este punto donde la política exterior encuentra su empalme con la ciencia, al tratar de aprovechar sus capacidades de establecer lazos comunes y definir proyectos conjuntos.

El esfuerzo por lograr la aceptación de ese término en el ámbito político demostró ser efectivo - respetando las variantes en la acentuación de esta iniciativa-, así que el gobierno de la gran coalición entre el partido democristiano y el socialdemócrata (2005-2009) lo adoptó como uno de los ejes de su proyección de Alemania en diferentes partes del mundo. Con el cambio de gobierno a una coalición entre el partido liberal y el partido demócrata-cristiano (2009-) el neologismo de Außenwissenschaftspolitik en términos de diplomacia científica, al parecer necesitó un rediseño en la descripción de los diferentes esfuerzos de los Ministerios de Educación e Investigación y Relaciones Exteriores respectivamente: se acogió el término de las "relaciones científicas internacionales", que se diferencia de alguna manera de un concepto alternativo que hubiera podido ser empleado en términos de una "política global científica". Es ahí donde se hace patente que esta nueva área de la acción externa del gobierno alemán parece estar sometida a las prioridades de la política interna y, por lo tanto, expuesta a los vaivenes de la composición del gobierno en sus diferentes etapas. Esto implica a su vez que se tenga que optar por la sostenibilidad de este esfuerzo, en caso de que se considere que puede ser objeto de diferentes coyunturas en la conformación de un área política que es de vital importancia para el posicionamiento de Alemania en el mundo.

\section{Retos para una política de relaciones científicas internacionales}

La política internacional científica se está moviendo en un campo muy competitivo en cuanto a la presencia en el mercado y la influencia, respectivamente, en el poder internacional. Es ahí donde se articulan los objetivos centrales, ya sea en tanto que se quiera garantizar el acceso a recursos de carácter global o atraer talentos, apoyo y aceptación para el sistema científico alemán y en sus respectivos resultados. Esta situación de competencia internacional por "las cabezas" pone de manifiesto que Alemania entra al cuadrilátero como país individual, sin duda en el marco de su nexo europeo, pero teniendo en mente 
también la competencia en el ámbito de la Unión Europea (Maihold 2007). Se busca potenciar el soft power (Perthes 2007), concepto presente en la teoría de las relaciones internacionales, cuya finalidad es la de ubicar los elementos de atracción y de ejemplo como incentivos para posicionar un país en las relaciones internacionales. Para Alemania, que como país no dispone de muchos recursos de hard power, es esencial recurrir a esta proyección de poder a nivel internacional (Wittig 2007). La diplomacia científica, en ese sentido, aporta a lo que ha ido definiéndose como smart power la capacidad de combinar hard and soft power en una estrategia exitosa. Sin embargo, esta intencionalidad todavía no perfila la decisión que se toma frente a la disyuntiva de doble uso que podría desprenderse de este planteamiento: por un lado una visión que enfatiza la utilidad de la ciencia para los esfuerzos diplomáticos, mientras que por el otro lado se busca reforzar el apoyo de la diplomacia para la ciencia. Sin querer recurrir a posiciones que realcen una diferencia de características fundamentales de las dos áreas y que por lo tanto presuponen una cierta posición de incompatibilidad de sus lógicas internas de acción, no hay que olvidar que el interés de la política por encontrar una utilidad en otros sectores de intercambio -en este caso académico-implica una pérdida de independencia en su accionar, el cual se encuentra sometido a las lógicas que la política como tal trata de proyectar para sus actores y su clientela internacional (Mak 2010). Esto sale a relucir, cuando se relaciona este debate con aquel de los países que son de mayor interés en el posicionamiento de Alemania en el mundo. Es decir, las naciones emergentes y de reciente industrialización, llamadas "Gestaltungsmächte" o poderes constructivos, que se han convertido en los objetos-meta de una cantidad de esfuerzos políticos que abarcan tanto la política económica, como la política científica, sin excluir la política global (Auswärtiges Amt 2011). Sin embargo, aún no se han logrado descubrir las aportaciones específicas de la diplomacia científica, ya que hasta la fecha no hay evaluaciones sobre este tipo de relacionamientos de los que se han servido los diferentes países.

\section{Problemas en la conformación de una diplomacia científica}

La conformación de una diplomacia científica tiene que enfrentar una cantidad considerable de problemas, por el simple hecho de que es un proyecto de carácter transversal entre los diferentes ministerios y sus respectivas agencias, lo que implica lidiar con la disparidad organizacional de los actores involucrados. Ellos no solamente introducen sus propias lógicas de acción en el momento de formar parte de las agencias de implementación, sino que incluyen también aquellas instituciones que tratan de participar en la definición y la 
priorización de las decisiones a tomar. El simple hecho de un cierto traslape entre diferentes áreas políticas (como son la política exterior y la política científica) multiplica los problemas y las expectativas, que no solamente se refieren a los intereses de participación en estos procesos de definición política, sino que también incluyen las estrategias organizacionales propias dentro de la administración pública y las correspondientes agencias encargadas.

Por lo tanto, en este campo tiende a reproducirse cierta tensión entre la cooperación y la competencia, la cual no solamente se expresa a nivel nacional, sino que también es extensible a nivel internacional. Ante la escasez de esfuerzos multilaterales de investigación (Schavan 2008: 32), la Unión Europea ha ido articulando un claro interés por posicionarse en las políticas internacionales de carácter científico.. De esto deriva cierta rivalidad entre el nivel regional y nacional que también se transfiere a las contrapartes allende del espacio europeo, que de pronto tienen que elegir entre diferentes propuestas que les ofrecen las instancias nacionales europeas, sin que haya habido una coordinación previa por parte de las instituciones en los países miembros de la UE. Este aspecto de competencia nacional dentro del marco europeo desorienta a las universidades e instituciones de investigación que, ante la multiplicidad de ofertas en el mercado, tienen gran dificultad para decidirse por una.

Las tensiones que emanaron de allí al final de cuentas no coadyuvaron a mejorar la posición alemana y europea en los espacios internacionales; inquietudes que se añaden a las complicaciones que resultan de las lógicas encontradas en el mismo sistema científico. Allí se enfrentan modalidades antagónicas de orientación e interacción social: por un lado existe el régimen (in)formal de los peers académicos, quienes actúan bajo el criterio de la evaluación científica y de los méritos alcanzados por los investigadores, mientras que por el otro lado las agencias de intercambio y promoción científicos proceden orientados en criterios formales de organización, autoridades y jerarquías, en los que quieren ver implementados sus prioridades y planes de acción, en la mayoría acordados con sus instituciones contraparte en los países prioritarios. Estas lógicas no necesariamente llegan a coincidir, por lo que dejan espacio y oportunidad para interferencias de carácter político.

Esta particularidad no sólo define la zona de contacto entre política y ciencia, sino que pone en evidencia una relación problemática gracias a la que podría llegar a utilizarse la ciencia en favor de los intereses de la política exterior; por ejemplo, la definición de las naciones-meta, que en el caso alemán son determinadas como países emergentes, se orienta en el interés político de fortalecer las relaciones con los países tipo BRIC, codiciados por los diferentes actores internacionales en esta materia. Aparte se está desarrollando todo el debate sobre la cooperación académica en términos de aportaciones al desarrollo de países subdesarrollados en el marco de los Objetivos del Milenio (Schütte 2007: 
33), ya sea a través de la conformación de estructuras idóneas para la investigación propia, la capacitación del personal académico o la formación de condiciones para que funjan como incubadoras técnicas y tecnológicas adecuadas.

\section{Experiencias internacionales: Inglaterra y EE.UU.}

Aunque el deseo de lograr una mayor internacionalización de la ciencia y la tecnología se consuma en aras de una desnacionalización de los esfuerzos propios de cada Estado, el afán central sigue apuntando a los sistemas nacionales de innovación y su orientación para lograr una presencia nacional expandida en el ámbito internacional. La ausencia de agendas internacionales de innovación le confiere a esta situación una característica paradójica: una necesidad internacionalizada de adquisición del conocimiento por un lado y una administración nacional por el otro (Dolata 2006).

Aparte, es palpable que los intereses de cada país se desarrollan de una manera bastante variada: por un lado existen aquellos que anhelan atraer nuevos conocimientos desde el exterior e intentan lograr condiciones para su absorción, mientras que otros se consideran los portadores de estos conocimientos de innovación, por lo que tratan de posicionarse de tal manera que esta ventaja no caduque a corto plazo. Llama la atención que en este sentido exista un debate en algunos espacios que considera la internacionalización en ciencia e investigación como un juego de suma cero, en tanto sigue la lógica de que la ganancia de un país siempre va a costa de otros. Esta posición manejada en los países desarrollados que tratan de "defender" su avance en cierta área científica, devela también sus límites cuando es confrontada con los sistemas de producción de conocimiento nuevo, especialmente de compañías internacionales que tienen sus propias capacidades de investigación distribuidas en el ámbito internacional. Esta presencia transnacional logra conciliar las inversiones en $\mathrm{I}+\mathrm{D}$ como un elemento central de su posicionamiento internacional bajo el amparo de los derechos de protección de la propiedad intelectual. Por lo tanto, una perspectiva que se limita a observar a los actores públicos en su quehacer referido a la cooperación y el intercambio científico-tecnológico, sólo en parte será capaz de cubrir las dinámicas en este sector. Por eso, para impulsar un nuevo dinamismo al desarrollo tecnológico habrá que considerar de entrada la cooperación entre actores públicos y privados (public private partnership), especialmente de aquellos sectores cercanos a los avances concretos en los desarrollos punta.

Una revisión de las políticas de I+D pone de manifiesto que existen políticas que, desde un punto de vista tipológico, cubren los siguientes objetivos (Flink/Schreiterer/Turekian 2010): 
- Atracción de investigadores e inversiones extranjeros

- Mejoramiento del rendimiento del país en materia de investigación científica, desarrollo tecnológico, innovación y competitividad económica

- Nivelación del acceso al conocimiento que es distribuido globalmente y derribo de las barreras para el acceso a mercados de punta para la promoción de productos de alta tecnología y nuevos servicios

- Ponerse al tanto de las políticas de investigación, de fomento tecnológico y desarrollo económico de otros países

- Contar con el apoyo de la diplomacia científica para apoyarse en las experiencias ya hechas y establecer redes internacionales entre compañías, actores nacionales, universidades e institutos de investigación

Tal programa implica revertir la carencia de científicos y capacidades industriales que existen en materia de atracción u oferta a nivel nacional, aplicar el llamado brain-gain o tratar de romper el aislamiento que existe en cuanto a la integración en los procesos internacionales de generación de conocimiento; por lo tanto, es urgente aumentar la atracción de científicos foráneos, inducir procesos de reconversión en los aparatos industriales de investigación y desarrollo e integrarse más plenamente en los mercados centrales de desarrollo científico-tecnológico, no solamente a través del desarrollo de capacidades administrativas propias y del manejo científico, sino también por medio del establecimiento de redes internacionales entre empresas y científicos (Edler/Boekholt 2001: 314 s.). Es el camino que siguen los EE.UU. e Inglaterra, que se consideran parte de aquellos mercados centrales que generan el avance tecnológico y que asimismo tratan de mantener su posición como mercado líder.

Esta situación encuentra a veces su contrapeso en el momento en que los representantes de estos países no estén dispuestos a asumir una posición de reciprocidad en los arreglos de carácter bi- y multilaterales para el desarrollo de los sistemas de innovación con otros países, prefiriendo mas bien un control de sus adelantos y tratando de establecer mecanismos como public-private-partnerships de corte nacional. A este panorama, el gobierno brasileño le ha contrapuesto su "diplomacia de innovación" y ofrece, por ejemplo, la tecnología para biocombustibles bajo un esquema de igualdad y sostenibilidad (Dickson 2010). Sin embargo, también en este caso se prefiere proceder a través de un estricto bilateralismo, ya que se considera esta modalidad más compatible con el interés por manejar las relaciones exteriores de su país en un formato no demasiado abierto.

Las lógicas presentes en estos contextos son dinámicas que buscan inducir un aprendizaje organizacional hacia adentro y se diferencian de aquellas experiencias que generan efectos de aprendizaje volcándose hacia afuera al esta- 
blecer esquemas de innovación a través de redes con formatos de cooperación transnacional. La competencia a la cual hoy en día están expuestos los sistemas nacionales de innovación tienen características globales y, por lo tanto, en el contexto de la Unión Europea (UE), existe la necesidad de plantearse acciones comunes como lo muestra el European Research Council. Estos esfuerzos, sin embargo, no han tenido hasta la fecha el efecto deseado: no se han logrado encontrar procedimientos adecuados que faciliten una mayor coherencia con las estrategias nacionales. Los instrumentos de la cooperación científica europea se asemejan demasiado a los formatos utilizados en los espacios nacionales, dejando de alguna manera en suspenso la agenda acordada para que se dé la confluencia de los programas manejados por ministerios nacionales.

Ahí desempeña también un papel central el debate sobre la correspondencia entre los esfuerzos nacionales y los intereses de fomento desde las políticas de cooperación al desarrollo, en tanto que los países del Sur se podrían convertir en contrapartes para la construcción de nuevas redes internacionales en materia de investigación y desarrollo. La creación de un área europea de investigación y su compatibilidad con los compromisos asumidos en el marco de los Objetivos del Milenio son retos centrales de la política externa de la misma UE, que ha recurrido a un procedimiento de coordinación de políticas para lograr la conciliación interna de sus prioridades con resultados mixtos. En el marco organizacional del fomento a la ciencia y a la tecnología (Bučar 2010: 21) se pone de relieve que, hacia adentro de la misma institucionalidad europea, existen muy diferentes conceptos que, si bien no han entrado en una contradicción abierta, hasta la fecha no han logrado desplegar todos los potenciales que podrían haberse obtenido con base en la confluencia de los sistemas.

El reto que también plantean hoy en día ciertas empresas internacionales para el avance tecnológico ( global challenges) en América Latina destaca en el discurso que se ha ido generalizando sobre las multilatinas (Santiso 2008) que han logrado una posición preponderante entre un conjunto de empresas con potencial para ocupar un papel de líder a nivel mundial. Aunque a veces se les ha asociado a intereses de explotación de recursos naturales, existen también aquellas que han asumido un claro liderazgo en el sector industrial o de servicios, de manera que no debemos subestimar su capacidad de impactar sobre la generación de valor agregado (Verma et al. 2011).

\section{Inglaterra: ¿política a costa de la ciencia?}

Si concentramos nuestra atención en el caso inglés, éste podrá ser muy aleccionador: en los pasados diez años Inglaterra ha desarrollado una diplomacia científica que se guiaba-siguiendo el ejemplo de que el sistema como tal no 
es capaz de orientar otras áreas políticas- en la agenda gubernamental para el cambio global (Millenium Agenda for Global Change), publicada en el año 2000, que servía para poner en operación los objetivos del milenio. Asimismo, la Oficina de Relaciones Exteriores tomó la iniciativa de fomentar la investigación y tecnología a través de la inauguración de una red de diplomáticos "científicos" en ciertas áreas centrales, con el fin de aumentar y expandir la presencia del Reino Unido en todo el mundo. Este impulso proveniente de la política tuvo como efectos secundarios la matización de los intereses específicamente diplomáticos de soft power con base en la agenda política y su disposición de proyección, dejando de lado las necesidades propias de la comunidad científica. Esta situación tuvo como efecto que la misma representación de los científicos, los Research Councils United Kingdom (RCUK), lanzara su propia estrategia de internacionalización y abriera oficinas de representación en Bruselas, Washington, Beijing y Delhi. ${ }^{1}$

Sin embargo, las dificultades de coordinación que sufrieron los diferentes intentos de impulsar la presencia británica en materia de ciencia e innovación se refleja en el esquema que se creó para facilitar la interacción de los diferentes ministerios que se sumaron a este esfuerzo a través de una plataforma de coordinación llamada "Global Science and Innovation Forum” (GSIF 2006), fundada en 2006 y cuya labor es la de fungir como vehículo para facilitar la información y el intercambio con el fin de coordinar el papel que debe jugar el Reino Unido en materia de cooperación científica y de innovación. El esfuerzo del GSIF y su posible éxito se debe a sus capacidades de articular en un alto nivel político del gobierno británico a los diferentes actores, representado en la institución que asesora el gobierno en materia política como lo es el Government Chief Scientific Adviser. El hecho de ser la persona que asesora al primer ministro en materia de ciencia, ha creado además una constelación a través de la cual pueden llegar a reconciliarse los diferentes intereses estratégicos entre las representaciones de los ministerios por un lado y, por el otro lado, se logra generar un estatus de coordinación que no corra el peligro de estar perdiendo su efectividad cuando surjan dificultades de interacción interinstitucional (Flink/Schreiterer 2010: 671 s.)

\section{EE.UU: en busca de un camino para la diplomacia científica}

Con la Asociación Americana para el Avance de la Ciencia (American Asociation for Advancement of Science, AAAS) se ha conformado, ya desde el siglo

\footnotetext{
$1<\mathrm{http} / / /$ www.rcuk.ac.uk/documents/framework/framework.pdf $>(17.11 .2011)$
} 
XIX, una instancia central para la organización del sector científico y su proyección hacia el exterior en los EE.UU. La AAAS mantiene un Centro para Diplomacia Científica ${ }^{2}$ a través del cual se hace el intento de utilizar la cooperación científica para la promoción del entendimiento y prosperidad internacionales. Para tal efecto, la AAAS aumentó su presencia territorial en amplias partes del mundo a través del envío de science envoys, destacadas personalidades de la ciencia que se dedican a promover la presencia científica de los EE.UU. en el exterior. Igualmente, en el mismo Departamento de Estado, cada año se están colocando a jóvenes científicos cuya tarea es fungir como asesores en materia de política exterior con el fin de fortalecer las misiones diplomáticas de EE.UU. (Flink/Schreiterer 2010: 674).

La administración Obama ha incrementado este programa de enviar a destacados científicos como emisarios diplomáticos, especialmente a comunidades musulmanas, y las considera un new beginning, iniciativa con la que se tratan de reconstituir las relaciones de los EE. UU. con el Medio Oriente, África del Norte y el Sureste de Asia (Campbell 2009). Más allá de los esfuerzos bilaterales establecidos y la cooperación estadounidense en esfuerzos multilaterales de investigación, la iniciativa del presidente Obama corresponde a un uso explícito de la ciencia como instrumento para restablecer relaciones con países que se han encontrado en una cierta situación de aislamiento en cuanto a sus relaciones políticas. Por lo tanto, puede considerarse un paso para iniciar un dialogo, lograr intercambios y, a lo mejor, una cierta colaboración en un sector donde las medidas diplomáticas clásicas son poco eficaces. Así, es más que lógico que para este tipo de programas se tomen en consideración retos de carácter global como la alimentación, el agua, la energía, el clima, etc., para cuya solución se quieren aportar conocimientos tecnológicos y técnicos con la intención de establecer además relaciones de asociación con colegas científicos en esos países meta.

Para tal efecto, se quiere recurrir al lenguaje común de los científicos, dejando a un lado en un primer momento los temas que podrían prestarse para un distanciamiento entre los países y para suscitar conflictos entre sus posicionamientos políticos. La pregunta de si la diplomacia científica realmente presenta una situación de win-win con respecto a la cooperación científica y su conexión con los intereses y motivos de la política gubernamental (Copeland 2011) es un debate abierto especialmente en los EE.UU. ya que se alega su relación precaria con respecto a ciertos países específicos. La expectativa política de que la ciencia pueda allanar el camino para entablar una cooperación científica con países tan disímiles como Pakistán, Irán, China, Corea del Norte o Libia, representa uno de los retos más debatidos en la actualidad en materia de política

\footnotetext{
${ }^{2}$ Center for Science Diplomacy: < http://diplomacy.aaas.org/> (12.04.2011).
} 
científica. El alcance que se pretende obtener a través del empleo de la ciencia para fines de la política exterior es muy evidente en una situación en la cual la política exterior estadounidense tiene una imagen muy negativa -especialmente al final de la era Bush-y esta preocupación afloraba no solamente a nivel político sino también universitario. El interés por fortalecer la presencia de la diplomática científica para fines de la política exterior estadounidense, quedó explícitamente manifiesto en un documento conjunto bipartidista que fue adelantado por representantes científicos y políticos del país en febrero de 2010, bajo el titulo "Science diplomacy is crucial to US foreign policy". El hecho de que este documento haya sido promocionado por el "Partnership for a Secure America" (2010) y avalado por el AAAS pone de manifiesto que la funcionalización de la ciencia para la realización de metas en política exterior sigue siendo un tema central. Más allá de este interés por suavizar las relaciones críticas a nivel político, también está presente la tendencia de mantener vigente la posición de EE.UU. como líder en el desarrollo científico-tecnológico, por lo cual el afán de relacionarse con personalidades de la avanzada en materia de investigación es un elemento central que se quiere garantizar para el futuro.

La participación de muchas instancias públicas en la presencia internacional de la ciencia y de la tecnología en el caso de los EE.UU. queda evidente en la existencia de un consejo presidencial de asesores en ciencia y tecnología, fundado a instancias del Departamento del Estado, que se ocupa de esta materia y de los impulsos en los diferentes ministerios, sin que se haya logrado hasta la fecha consolidar un Ministerio de Ciencias en este país. El Center for Science Diplomacy del AAAS pone de relieve que su mayor compromiso radica en establecer un nexo entre investigadores, analistas políticos y tomadores de decisión, con el fin de allanar el camino a la diplomacia científica en su función de catalizador entre sociedades y con el afán de fortalecer las interacciones de actores del ámbito de la sociedad civil (AAAS 2011).

La revisión de estos casos, a los que podríamos añadir unos cuantos más, pone en evidencia que el área de la promoción de relaciones científicas internacionales es reconocida en muchos países como una tarea central para que el respectivo país pueda mantener una presencia internacional significativa. Sin embargo, se ve que hay cierta disparidad entre actores y tareas, que no ha podido ser organizada en un esquema coherente que pudiera servir de modelo para su aplicación en otros países. Por el otro lado, los países y Estados meta son bastante disímiles porque, mientras Inglaterra trata de tomar en consideración a los Estados emergentes que funcionan como maquinas del crecimiento económico mundial, en los EE.UU., de alguna manera se ve el aporte de la ciencia en términos de reducir riesgos para sus relaciones exteriores con países que han presentado cierto problema en el trato de la política oficial. La configuración concreta siempre se mueve en una amplia gama de motivos: 
- el acceso a mercados prometedores y desarrollos en ciencia e investigación interesantes,

- acompañados de la promoción de los propios adelantos en esta materia y

- la promoción de las instituciones de la educación superior para un mercado demandante en estos países,

- un interés explícito por participar en el acceso a personal científico altamente capacitado

- para poder lograr por esta vía acaparar este personal capaz de alimentar el propio aparato científico.

\section{Acceso, promoción e influencia: lógicas de la diplomacia científica alemana}

El surgimiento reciente de la diplomacia científica en Alemania ha incitado el debate de si se trata de una conceptualización sistemática de un área política nueva o más bien de un acercamiento $a d$ hoc al cual le hacen falta ciertos elementos esenciales que dan sustancia y continuidad a la acción política. Tomando en consideración la transversalidad del interés político que lo anima, se ha planteado la pregunta de si la diplomacia científica puede considerarse un área política propia si se toma en cuenta la dificultad que cuesta definir el límite de esta área y las lógicas que la rigen. Con respecto a estas interrogantes se ha afirmado que el acceso a investigadores, a los resultados de sus investigaciones, a las respectivas instituciones, a los recursos naturales y al capital son esenciales para impulsar la capacidad de innovación y la competitividad nacionales. Además, la promoción de los logros del propio país en materia de I+D en el marco de su mercadeo global es una precondición para atraer a personas y compañías; asimismo, se desea ampliar la influencia individual con el compromiso de los ciudadanos y las organizaciones de la sociedad civil a nivel mundial, cruzando así las fronteras del propio país.

Ante esta descripción de tareas asociadas a la diplomacia científica, pueden generarse dudas de si el mismo servicio exterior está suficientemente preparado para asumir estas responsabilidades en la dirección de esta política; más allá de ello, habrá que discutir cuál o cuáles son las instituciones capaces de implementar estratégicamente estas iniciativas. Alemania cuenta con una amplia red de actores en esta área, empezando con la DFG Deutsche Forschungsgemeinschaft (Comunidad Alemana de Investigación) ${ }^{3}$, la Max-PlanckGesellschaft ${ }^{4}$ y otros centros de investigación extrauniversitarias, como la

\footnotetext{
$3<$ http://www.dfg.de $>$ (12.03.2011).

$4<$ http://www.mpg.de $>(12.03 .2011)$.
} 
Helmholtz-Gemeinschaft Deutscher Forschungszentren ${ }^{5}$ o la FraunhoferGesellschaft. ${ }^{6}$ En el área del intercambio académico, el Deutscher Akademischer Austauschdienst (DAAD; Servicio Alemán de Intercambio Académico) ${ }^{7}$, la Alexander von Humboldt Stiftung y los mismos secretarios para la ciencia en las Embajadas alemanas en el exterior son instancias que pueden facilitar programas y proyectos financiados por parte del gobierno federal. Sin embargo, estos actores no necesariamente juegan "en equipo" y logran complementar sus capacidades para alcanzar prioridades y objetivos comunes. Al mismo tiempo, la política no siempre ha sido capaz de definir claramente las regiones meta o las materias centrales para sus intereses estratégicos particulares o sus compromisos globales. Así, la expectativa puesta en estas instituciones de que se pueda a través de sus programas lograr una expansión global de normas y valores orientada en el desarrollo pacífico y la resolución de conflictos tiene limitaciones implícitas para surtir efectos.

Esto se vuelve evidente desde las mismas bases programáticas de los dos ministerios centrales en el área: mientras que el Ministerio de Educación e Investigación (BMBF) sigue el concepto de una política científica global, el Ministerio de Relaciones Exteriores más bien promueve una diplomacia científica para lograr un avance en el combate de problemas de alcance global, para formar capacidades en regiones de desarrollo y promover capacidades cívicas y el diálogo. En su documento base "Internationalisierungsstrategie für Wissenschaft und Forschung", del año 2008, el Ministerio de Educación e Investigación (BMBF 2008) define cuatro prioridades propias:

- Fortalecimiento de la cooperación científica con líderes globales

- Explotación internacional de potenciales de innovación

- Intensificación de la cooperación con países en vías de desarrollo en materia de educación, investigación y desarrollo a largo plazo

- Asumir responsabilidad internacional y resolver retos globales.

Para lograr estas metas se pretende formar a investigadores jóvenes con un perfil internacional en aras de ampliar la movilidad internacional y especialmente europea de esta nueva generación alemana. Al mismo tiempo, se desean coordinar mejor los programas de fomento a nivel nacional, regional e internacional para aprovechar de mejor manera las complementariedades. Cumpliendo con el interés de acercar la cooperación al desarrollo y la cooperación científico-tecnológica se aspira acordar agendas de prioridades con los países contrapartes.

$5<$ http://www.helmholtz.de $>(12.03 .2011)$.

$6<$ http://www.fraunhofer.de $>(12.03 .2011)$.

$7<$ http://www.daad.de $>(12.03 .2011)$. 
Finalmente, la implementación de una agenda internacional de investigación debería dar respuestas a los retos globales como el cambio climático, el problema de recursos naturales, las amenazas de seguridad y las enfermedades globales infecciosas, para orientar los programas multilaterales de fomento.

Operativamente se esperan mejores resultados con la presencia coordinada de las instituciones de la ciencia alemana con las representaciones de la industria alemana en el exterior. En concreto, se fomentan exposiciones y eventos de promoción conjuntos para desarrollar mayores complementariedades entre los dos sectores. Lo mismo se intenta a nivel europeo, en el marco de estrategias comunes con respecto a ciertos países contrapartes de acuerdo a la estrategia alemana de internacionalización. Ésta se someterá a una evaluación en intervalos fijos para poder medir su impacto sobre la realidad.

\section{El caso de las relaciones científicas entre Alemania y América Latina}

En las décadas de 1980 a 2000 se ha detectado una contracción masiva de solicitudes desde América Latina para becas de estudio en Alemania (Janetzke 2001). Esto vale específicamente para el área de ciencias sociales, derecho y estudios culturales, por lo que se nota una disminución de la tradicional cercanía de las humanidades para con la investigación en Alemania que data de los años 20 del siglo pasado. Por el otro lado se puede identificar una gran demanda de investigadores con doctorado en las universidades de América Latina, ya que los procesos de certificación de programas de estudio a nivel de maestrías y doctorados han aumentado la presión sobre las universidades de incrementar el número de académicos con doctorado en sus centros de estudio. Es sorprendente que esta dinámica no se haya reflejado oportunamente en las universidades de Alemania, lo cual de alguna manera se ha atribuido a la lengua alemana como barrera de acceso a los estudios de doctorado en universidades de este país. El alemán no parece fungir hoy en día como lengua franca de la comunicación académica, por lo que muchas universidades han optado por ofrecer cursos y estudios de posgrado en inglés. En consonancia con la estrategia de Bolonia de homologar los exámenes y estudios a nivel europeo, se espera que las universidades de Alemania se vuelvan más atractivas para estudiantes de América Latina.

Revisando las cifras de los estudiantes latinoamericanos en Alemania, se puede detectar, siempre dependiendo del país y tomando como base una situación constante y a veces ascendente, una tendencia positiva, que en el caso de Brasil, México y Colombia refleja mayores avances.

En el marco institucional de los acuerdos de cooperación entre universidades a escala mundial, solamente Brasil logra acercarse al nivel de Rusia y 
FIGURA 1

Estudiantes latinoamericanos en Alemania

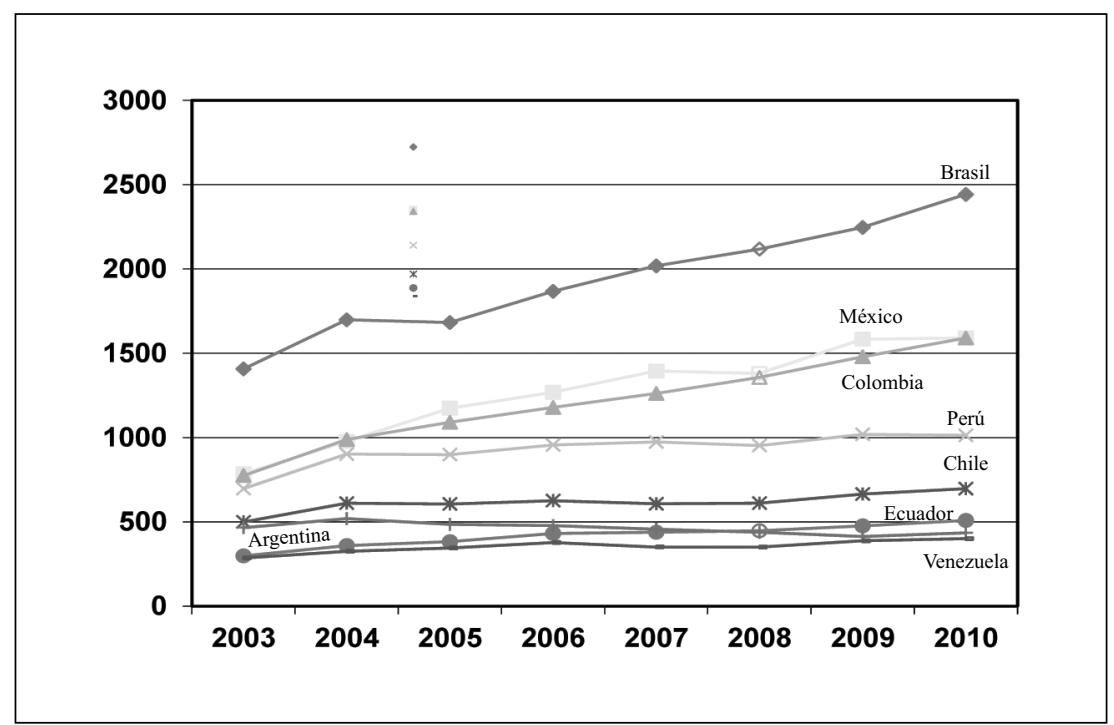

FUENTE: DAAD.

FIGURA 2

Asociaciones de universidades alemanas en el mundo

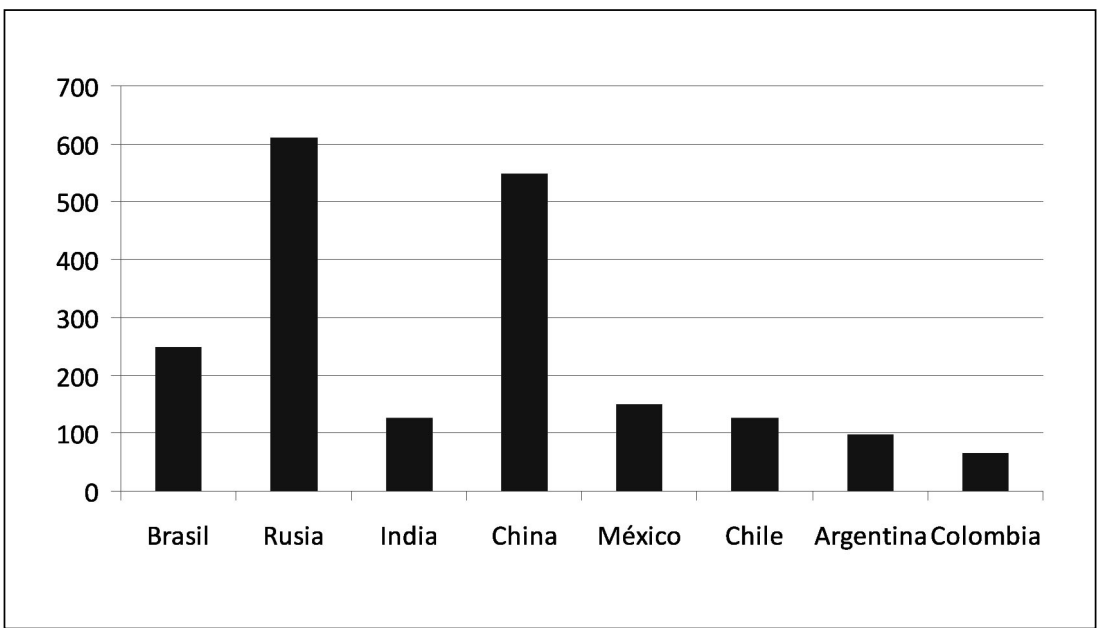

FUENTE: Wissenschaftskompass/HRK. 
China, mientras que India queda igualmente con un desarrollo relativamente bajo, al igual que México y Chile. Esta situación es sumamente sorprendente si uno ve en perspectiva histórica la larga historia de cooperación que ha existido entre universidades de Alemania y de América Latina. Como esta tradición académica data de muchos años atrás, hay que preguntarse por qué no ha logrado encontrar una expresión institucional correspondiente a tantas décadas de intercambio entre investigadores de los dos lados.

Es en este contexto en el que deben revisarse los alcances de la cooperación entre las respectivas entidades de fomento académico de los dos lados (CONICET/COLCIENCIAS/CONACYT/DFG), ya que a lo mejor un nuevo impulso debería pasar por otras entidades, como por ejemplo las asociaciones de universidades, los consejos de rectores u otras entidades similares.

El instrumento clásico de fomento en el intercambio académico es el otorgamiento de becas. Una revisión de los datos en el caso de la Alexander von Humboldt Stiftung pone de relieve que la participación de América Latina en la entrega de becas y premios ha tenido un desarrollo muy precario, logrando un avance del 3 al 4,6\% del total en una década.

En la distribución por países en América Latina se pone de manifiesto que son Brasil, Argentina y México los que han logrado un nivel más alto, de entre 4 y 7 becas al año, de una aplicación máxima de 18 solicitudes en el caso del Brasil. Esta situación pone de relieve que serán solamente los países con una estructura científica consolidada los que tengan posibilidades reales de ampliar su presencia a través de becas del sistema alemán.

Aparte de los elementos tradicionales (becas, invitaciones, programas de intercambio y de graduación), la política científica ha desarrollado actividades específicas para promover a Alemania en el mercado de la ciencia y de la tecnología de América Latina. Entre éstas hay que mencionar el Año AlemánBrasileño de Ciencia, Tecnología e Innovación 2010/2011, organizado por el BMBF y su contraparte, el Ministério da Ciência e Tecnologia do Brasil. Este evento trató de poner énfasis en las posibilidades de cooperación en la investigación en materia de medio ambiente, de sostenibilidad y del área marítima, que demostraron ser los ejes más interesantes para la colaboración. Además, en especial en el área de la investigación aplicada se enfocaron la tecnología de la información, la investigación de genomas, nano- y biotecnología, al igual que la salud y la aeronáutica. La fundación de un Fondo Común para la Ciencia representa en esta cooperación un nuevo elemento bilateral. Asimismo, en Brasil se inauguró en el año 2009 la Casa Alemana de Ciencia e Innovación, con sede en São Paulo (DIWH) ${ }^{8}$, apoyado por el Ministerio de Relaciones

\footnotetext{
$8<$ http://www.dwih.com.br/> (12.03.2011).
} 
FIGURA 3

Becas AvH solicitadas y concedidas 1994-1998

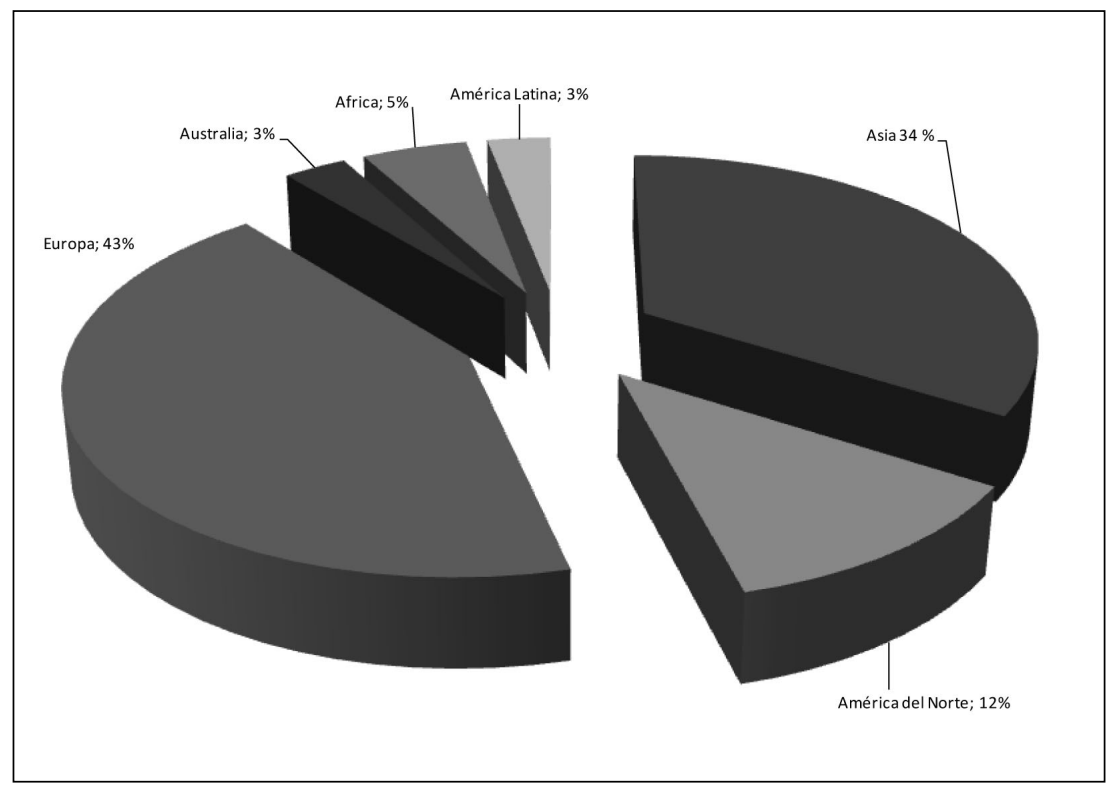

Becas AvH solicitadas y concedidas 2009

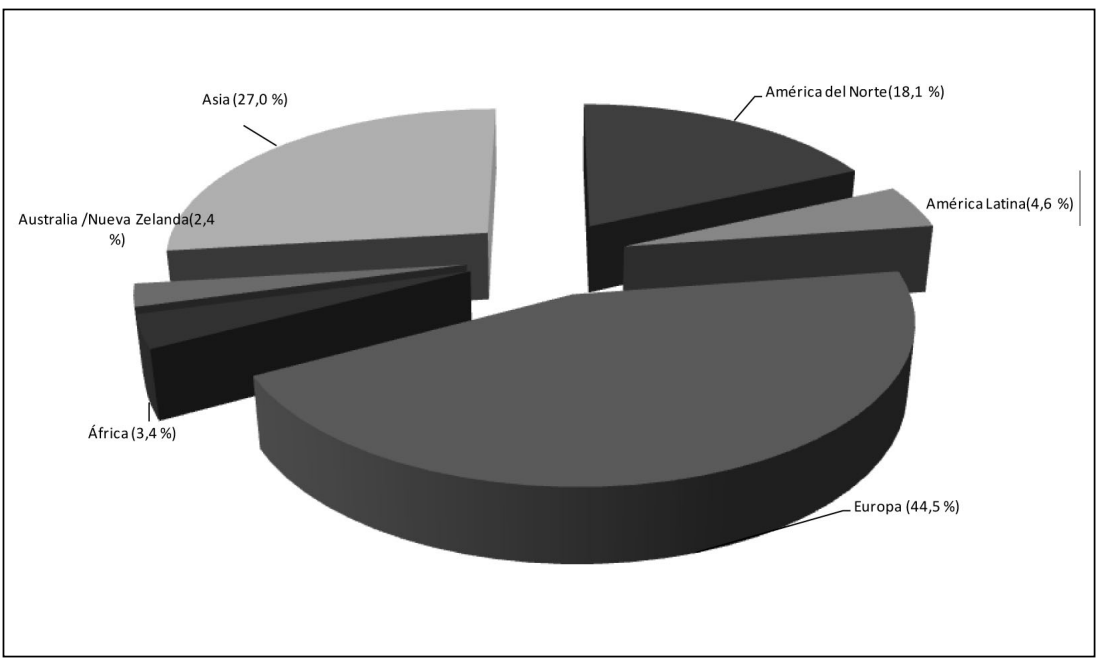

FUENTE: Jahresberichte Alexander von Humboldt Stiftung. 
Becas AvH 2008/2009 en América Latina por países

\begin{tabular}{|l|c|c|c|c|}
\hline \multirow{2}{*}{ Brasil } & \multicolumn{2}{|c|}{ 2008 } & \multicolumn{2}{c|}{2009} \\
\cline { 2 - 5 } & solicitadas & concedidas & solicitadas & concedidas \\
\hline México & 18 & 7 & 18 & 5 \\
\hline Argentina & 14 & 3 & 14 & 7 \\
\hline Chile & 6 & 6 & 9 & 4 \\
\hline Cuba & - & 5 & 5 & 2 \\
\hline Perú & 3 & - & 3 & 1 \\
\hline Costa Rica & - & - & 1 & 0 \\
\hline Bolivia & 1 & - & - & - \\
\hline Colombia & 2 & 1 & - & - \\
\hline Ecuador & 1 & 1 & - & - \\
\hline Honduras & 1 & - & - & - \\
\hline Venezuela & 1 & 1 & - & - \\
\hline
\end{tabular}

FUENTE: Jahresberichte Alexander von Humboldt Stiftung.

Exteriores y el BMBF con el interés de fomentar la interrelación de universidades e institutos de investigación de ambos países; que los científicos estén mirando con alguna preocupación la cercanía muy grande a intereses comerciales refleja de nuevo la relación precaria entre intereses políticos, económicos y científicos (Dungen 2011: 45). El Ministerio alemán de Relaciones Exteriores, por su lado, asignó al Heidelberg Center en Santiago de Chile 9 y a la Universidad del Norte, Sede Santa Marta/Colombia ${ }^{10}$ la calidad de Centros de Excelencia para Investigación y Enseñanza en colaboración con universidades alemanas. Esta variante concuerda con el creciente interés de "exportar" las ofertas de enseñanza hacia América Latina y el Caribe, a veces con dependencias completas al estilo de las universidades de los EE.UU. (Werz 2006).

\footnotetext{
$9<$ http://www.heidelberg-center.uni-hd.de/> (12.03.2011).

$10<\mathrm{http}: / /$ www.cemarin.org/> (12.03.2011).
} 


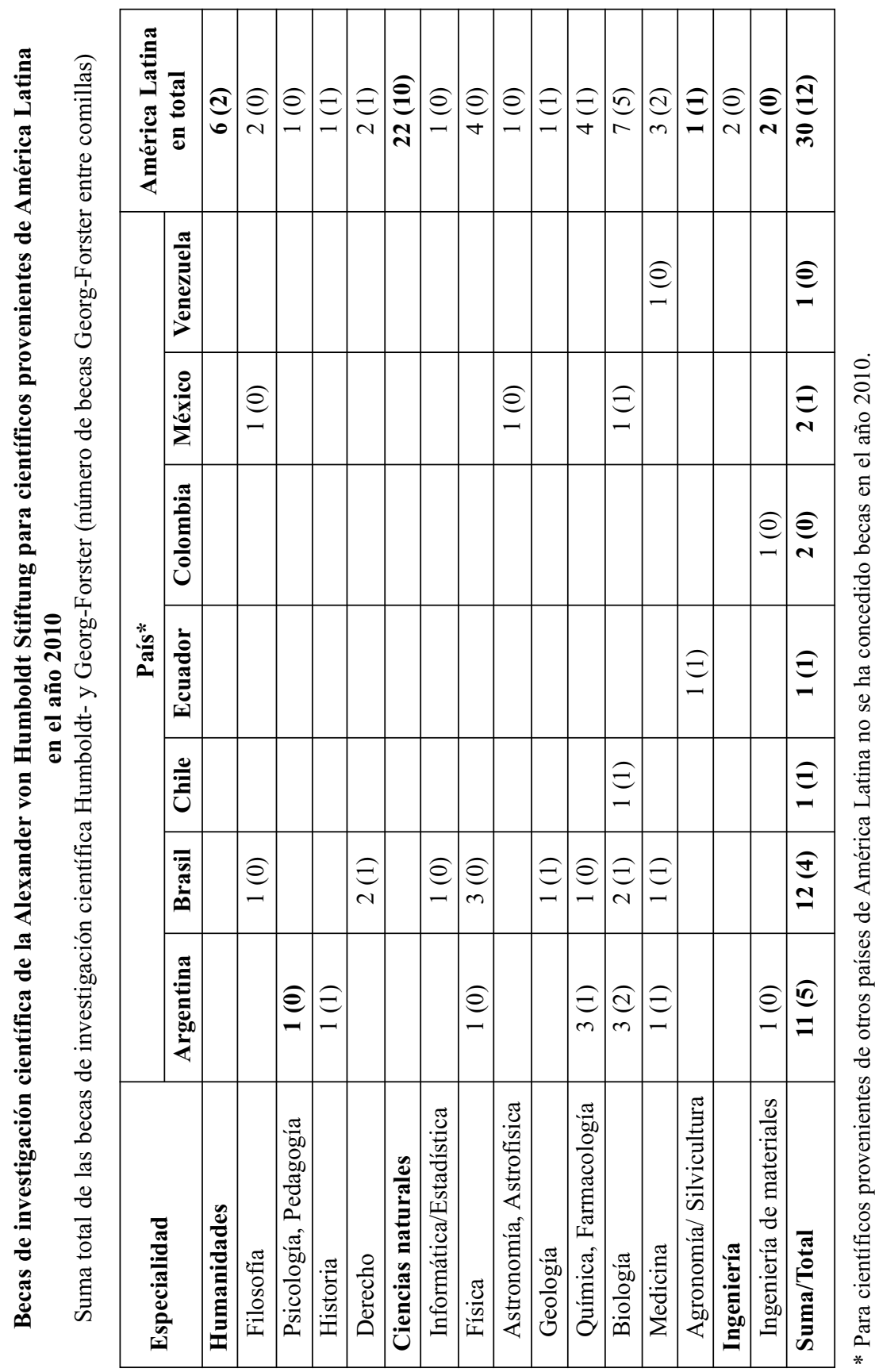


Finalmente, se ha diseñado un proyecto muy innovador con base en el Centro Universitario Alemán-Argentino (CUAA-DAHZ), ${ }^{11}$ con sede en Buenos Aires, que se encuentra en proceso de construcción y pretende fungir como aglutinador de proyectos bilaterales en el marco del Programa Binacional para el Fortalecimiento de Redes Interuniversitarias Argentino-Alemanas. Se aspira a promover y desarrollar programas de grado y posgrado entre universidades de ambos países que conlleven al establecimiento futuro de carreras binacionales con doble titulación. En el campo de la investigación se busca estimular y fortalecer la asociación académico-científica, alentando el intercambio de docentes, investigadores y alumnos. Aparte, siguen trabajando Cátedras especiales como la Cátedra Guillermo y Alejandro de Humboldt en la UNAM y el Colegio de México ${ }^{12}$, la Cátedra Walter Gropius en la Universidad de Buenos Aires/Argentina ${ }^{13}$ y la Cátedra Martius de Estudos Alemães e Europeus en la Universidad São Paulo/Brasil ${ }^{14}$ financiadas por parte del DAAD.

\section{Conclusiones}

La diplomacia científica como instrumento para restituir las relaciones académicas y de investigación entre Alemania y América Latina se ha propuesto una amplia gama de objetivos. Esta situación implica eminentes esfuerzos para corresponder a los grandes retos planteados, especialmente en tiempos de presupuestos reducidos y limitadas posibilidades a nivel de fomento académico ante una situación de mucha competencia. Sin embargo, la importancia asignada a la I+D debería también encontrar su expresión en América Latina, especialmente ante el declarado interés del gobierno alemán por impulsar su relación con la región (Auswärtiges Amt 2010) Aunque pueda haber mucha voluntad política (Maihold 2010), dependerá de los mismos actores en el área de la ciencia e investigación si se logran construir puentes y desarrollar formatos asociativos y de colaboración efectivos acordes a sus intereses. La política, por su lado, puede facilitar el acceso y la promoción, no solamente por sus objetivos de responsabilidades compartidas y beneficios mutuos en materia global, sino también en la movilización de intereses concretos articulados en las universidades y la industria. Para tal efecto, la formación de alianzas estra-

\footnotetext{
$11<\mathrm{http}: / /$ cuaa-dahz.org/es/home/> (12.03.2011).

$12<$ http://www.colmex.mx/index.php?option=com_content $\& v i e w=$ article $\& i d=$ $88 \&$ Item $=\&$ Itemid $=100>(23.03 .2012)$.

$13<\mathrm{http} / / / \mathrm{www}$.academicas.daad.org.ar/catedra-walter-gropius/> (23.03.2012).

$14<\mathrm{http} / / \mathrm{cm}$.daad.net/index.php?option=com_content\&view $=$ article\&id=61\&Itemid=28\&lang $=$ de $>(23.03 .2012)$.
} 
tégicas más allá de un afán declarativo podría aterrizar de una manera muy concreta en materia de ciencia buscando construir faros que puedan dar orientación a amplios grupos e instituciones del mundo académico tanto en Alemania como en América Latina. Que estos esfuerzos tengan que emprenderse en un ámbito de competencia internacional por el reclutamiento de las "mejores cabezas" y por las mejores ofertas bajo el criterio de la mayor atracción de las condiciones para la investigación y el desempeño profesional, implica un desafío especial para los sistemas de innovación, en Alemania y en América Latina.

\section{Bibliografía}

Auswärtiges Amt (2009a): "Wissenswelten verbinden. Deutsche Außenpolitik für mehr Bildung, Wissenschaft und Forschung". Berlin, Konferenzdokumentation Berlin 19-20 de enero de 2009, en: <http://www.auswaertiges-amt.de/cae/servlet/ contentblob/365322/publicationFile/3669/ProgrammDownload.pdf $>$ (20.04.2011).

- (2009b): "12 worlds of knowledge". Frankfurt/Berlin, en: $<$ http://www.auswaertigesamt.de/cae/servlet/contentblob/474658/publicationFile/3641/AWPTestimonials.pdf $>$ (10.03.11).

- (2010): Alemania, América Latina y el Caribe. Concepto del Gobierno federal alemán sobre América Latina y el Caribe. Berlin.

- (2011): Globalisierung gestalten - Partnerschaften ausbauen - Verantwortung teilen. Konzept der Bundesregierung. <http://www.auswaertiges-amt.de/cae/ servlet/contentblob/608384/publicationFile/165779/Gestaltungsmaechtekonzept.p df;jsessionid=731E0B38F02CA818516A81DD9E8E425A > (23.02.12).

BMBF (2008): "Strengthening Germany's role in the global knowledge society. Strategy of the Federal Government for the Internationalization of Science and Research", en: <http://www.bmbf.de/pubRD/Internationalisierungsstrategie-English.pdf $>$ (12.03.2011).

BUČAR, Maja (2010): Science and Technology for Development. Coherence of the Common EU R\&D Policy with Development Policy Objectives. Bonn: DIE.

CAmpbell, Cathy (2009): "Send in the Scientists. Why Mobilizing America's Researchers Makes Sense for Diplomacy", en: <http: //www.scienceprogress.org/ 2010/10/send_scientists/> (10.04.2011).

Copeland, Daryl (2011): “Science Diplomacy: New Day or False Dawn”, en $<\mathrm{http}$ :// www.themarknews.com/articles/5672-science-diplomacy-new-day-or-false-dawn> (12.03.2011).

DiCKSON, David (2010): “Innovation diplomacy: an alternative concept", en: <http:// scidevnet.wordpress.com/2010/06/27/innovation-diplomacy-an-alternative-concept> (12.03.2011).

DolatA, Ulrich (2006): “Technologie- und Innovationspolitik im globalen Wettbewerb. Veränderte Rahmenbedingungen, institutionelle Transformationen und poli- 
tische Gestaltungsmöglichkeiten”, en: Zeitschrift für Politikwissenschaft, vol. 16, $\mathrm{n}^{\mathrm{o}} 2$, pp. 427-455.

Dungen, Johannes von (2011): Vom Freund zum Partner: Die deutsch-brasilianischen Kulturbeziehungen im Wandel. Stuttgart: ifa.

EDLER, Jakob/BoeKholt, Patries (2001): "Benchmarking national public policies to exploit international science and industrial research: a synopsis of current developments", en: Science and Public Policy, vol. 28, n 4, pp. 313-321.

FLINK, Tom/SchreITERER, Ulrich (2010): "Science diplomacy at the intersection of S\&T policies and foreign affairs: toward a typology of national approaches", en: Science and Public Policy, vol. 37, November, pp. 665-677.

FLINK, Tom/SchreITERER, Ulrich/Turekian, Vaughan (2010): Towards better Approaches in Science Diplomacy - Communication, Coordination and Leadership. Berlin: WZB (mimeo).

Global Science And InNOvation Forum (2006): A Strategy For International Engagement in Research and Development, London (URN 06/1862), en: <http:// webarchive.nationalarchives.gov.uk/+/http://www.berr.gov.uk/files/file34726.pdf> (23.02.2012).

Janetzke, Gisela (2001): "Alexander von Humboldt, die Stiftung und ihre Wissenschaftsbeziehungen mit Lateinamerika", en: Maihold, Günther (ed.): Ein „freudiges Geben und Nehmen"? Stand und Perspektiven der Kulturbeziehungen zwischen Lateinamerika und Deutschland. Frankfurt: Vervuert, pp. 33-42.

MAIHOLD, Günther (2007): “Außenwissenschaftspolitik - zur Produktivität eines neuen Politikfeldes”, en: Alexander von Humboldt Stiftung (ed.): Außenwissenschaftspolitik - Wissenschaftsaußenpolitik. Bonn: Arbeits- und Diskussionspapier 7, pp. 16-20.

- (2010): Das neue Lateinamerikakonzept der Bundesregierung: Politikinnovation durch Konzeptentwicklung? Berlin: Ibero-Analysen $n^{\circ} 23$.

MAK, Stephen (2010): Science Diplomacy: Applying Science and Innovation to International Challenges. Wilton Park: Report on Wilton Park Conference 1037.

NetTElbeCK, Joachim (2008): "Wissenschaftsaußenpolitik - Asymmetrie der Wissensordnung und Orte der Forschung", en: Schütte, Georg (ed.): Wettlauf ums Wissen. Außenwissenschaftspolitik im Zeitalter der Wissensrevolution. Berlin: Berlin University Press, pp. 112-119.

PARTNERShIP FOR A SECURE America (2010): "Science Diplomacy is Crucial to U.S. Foreign Policy", en: <http: //www.psaonline.org/article.php?id=620> (10.04.2011).

Perthes, Volker (2007): "Soft Power als Teil der Auswärtigen Politik", en: Alexander von Humboldt Stiftung (ed.): Außenwissenschaftspolitik - Wissenschaftsaußenpolitik. Bonn: Arbeits-und Diskussionspapier 7, pp. 5-7.

SANTISO, Javier (2008): "La emergencia de las multilatinas", en: Revista de la CEPAL, vol. 95, agosto, pp. 7-30.

SchaVAn, Annette (2008): "Von Alexander von Humboldt lernen", en: Schütte, Georg (ed.): Wettlauf ums Wissen. Außenwissenschaftspolitik im Zeitalter der Wissensrevolution. Berlin: Berlin University Press, pp. 31-34.

SCHÜTTE, Georg (2006): "Diplomatie der Forscher. Wenn Deutschland international mithalten will, braucht es eine Außenwissenschaftspolitik. Ein Plädoyer”, en: Die Zeit, 12 de abril de 2006. 
SCHÜtTE, Georg (2007): “Außenwissenschaftspolitik - Wissenschaftsaußenpolitik: eine Querschnittsperspektive internationaler Politikgestaltung in modernen Wissensgesellschaften", en: Alexander von Humboldt Stiftung (ed.): Außenwissenschaftspolitik - Wissenschaftsaußenpolitik. Bonn: Arbeits- und Diskussionspapier 7, pp. 28-39.

- (2008): "Wettlauf ums Wissen: Außenwissenschaftspolitik als Herausforderung moderner Wissensgesellschaften. Eine Einführung”, en: Schütte, Georg (ed.): Wettlauf ums Wissen. Außenwissenschaftspolitik im Zeitalter der Wissensrevolution. Berlin: Berlin University Press, pp. 12-27.

SteINMEIER, Frank-Walter (2008): "Partner gewinnen und Potenziale nutzen - Warum sich Außenpolitik für Wissenschaft einsetzt", en: Schütte, Georg (ed.): Wettlauf ums Wissen. Außenwissenschaftspolitik im Zeitalter der Wissensrevolution. Berlin: Berlin University Press, pp. 28-30.

The Royal Society (2010): New Frontiers in Science Diplomacy. Navigating the Changing Balance of Power. London: RS Policy document 01/10.

Verma, Sharad et al. (2011): Companies on the Move. Rising Stars from Rapidly Developing Economies are Reshaping Global Industries. Boston: The Boston Consulting Group.

WERZ, Nikolaus (2006): “Von 'geborenen Partnern zu entfernten Verwandten'? Lateinamerika in der neueren deutschen Außenkulturpolitik", en: Lateinamerika Analysen, vol. 15, n 3, pp. 105-124.

WitTIG, Peter (2007): "Die Rolle und das Verständnis von Soft Power in der deutschen Außenpolitik", en: Alexander von Humboldt Stiftung (ed.): Außenwissenschaftspolitik - Wissenschaftsaußenpolitik. Bonn: Arbeits- und Diskussionspapier 7, pp. 8-10. 
